ISSN 0103-9954

\title{
CARACTERIZAÇÃO MECÂNICA E CONTRAÇÃO DA MADEIRA DE Sclerolobium paniculatum Vogel CULTIVADO EM UM PLANTIO HOMOGÊNEO SOB DIFERENTES NÍVEIS DE ADUBAÇÃO
}

\author{
MECHANICAL CHARACTERIZATION AND SHRINKAGE OF Sclerolobium paniculatum Vogel \\ WOOD IN A HOMOGENEOUS PLANTING UNDER DIFFERENT LEVELS OF FERTILIZATION
}

\author{
Joselito Bonifácio Oliveira ${ }^{1}$ Ailton T. Vale ${ }^{2}$ José Teodoro de Melo ${ }^{3}$
}

\section{RESUMO}

O objetivo do presente trabalho foi caracterizar a madeira de Sclerolobium paniculatum Vogel, de 18 anos de idade, oriunda de plantio homogêneo sob diferentes níveis de adubação localizado na EmbrapaCerrados, DF, quanto à contração e às características mecânicas (flexão estática, compressão paralela às fibras e cisalhamento). Não foram encontradas diferenças significativas entre as médias das variáveis estudadas que apresentaram os seguintes valores: resistência máxima à flexão estática $\left(f_{b}=650 \mathrm{~kg} / \mathrm{cm}^{2}\right)$, módulo de elasticidade $\left(E=59.877 \mathrm{~kg} / \mathrm{cm}^{2}\right)$, resistência máxima à compressão axial $\left(f_{c}=296 \mathrm{~kg} / \mathrm{cm}^{2}\right)$ e resistência máxima ao cisalhamento $\left(f_{v}=131 \mathrm{~kg} / \mathrm{cm}^{2}\right)$. Para cisalhamento e compressão axial, a testemunha apresentou o maior valor absoluto para MOR. A madeira de Sclerolobium paniculatum apresentou um elevado número de galhos, implicando uma elevada quantidade de nós. Os tratos culturais em plantios homogêneos de Sclerolobium paniculatum devem prever a retirada de galhos para produzir madeira de qualidade superior.

Palavras-chave: caracterização; madeira; Slerolobium paniculatum.

\section{ABSTRACT}

The purposa of this work was to study the influence of fertilization on wood quality of Sclerolobium paniculatum Vogel. A homogeneous planting trial, under different levels of liming and phosphorus, was established by Embrapa-Cerrados 18 years ago in Planaltina, Distrito Federal, Brazil, tropical wood savanna region. Mechanical tests conducted were static bending, parallel compression to grain, shear strength and shrinkage. No significant differences were observed for mechanical properties or for shrinkage, which presented: $f_{b}=650 \mathrm{~kg} / \mathrm{cm}^{2}, E=59.877 \mathrm{~kg} / \mathrm{cm}^{2}, f_{c}=296 \mathrm{~kg} / \mathrm{cm}^{2}$ e $f_{v}=131 \mathrm{~kg} / \mathrm{cm}^{2}$. Control treatment showed highest values for shear strength and compression parallel to grain. Too many branches in all trees and also too many knots in lumber were observed. Pruning is recommended for homogeneous planting of Sclerolobium paniculatum to avoid knots in order to be produced wood of superior quality.

Key words: characterization; wood; Slerolobium paniculatum.

\section{INTRODUÇÃO}

O pouco conhecimento do potencial de certas espécies lenhosas como fator de desenvolvimento econômico, social e ambiental na região do Cerrado brasileiro causaram danos ao meio ambiente, às vezes, irreversíveis que certamente poderiam ter sido evitados se as espécies do bioma fossem devidamente estudadas, de forma a permitir uma opção de uso do solo, competindo com a agropecuária e mineração. Os estudos sobre o potencial produtivo das espécies nativas do Cerrado brasileiro são escassos e recentes (Klink, 1996; Chévez Pozo, 1997; Rodrigues et al., 2002).

Uma das espécies com grande potencial para reflorestamento e de múltiplos usos na região do Cerrado brasileiro é o Sclerolobium paniculatum Vogel, conhecido como carvoeiro. Essa espécie é uma leguminosa nativa da região que apresenta elevada produção de biomassa, com madeira amplamente utilizada pelas comunidades rurais na construção civil, na marcenaria e como fonte de energia. É capaz de estabelecer-se numa ampla faixa de condições edáficas e de altitude, apresentando duas variedades vicariantes, a rubiginosum, comumente encontrada em cerradões e a variedade subvelutinum, mais freqüente

1. Engenheiro Florestal, MSc., Banco Nacional de Desenvolvimento Econômico e Social, Setor Bancário Sul, Conjunto 1, Bloco E, $13^{\circ}$ andar, CEP: 70076-900, Brasília (DF). josel@bndes.gov.br

2. Engenheiro Florestal, Dr., Professor Adjunto do Departamento de Engenharia Florestal, Universidade de Brasília, CEP: 70910-900, Brasíla (DF). ailtontv@unb.br

3. Engenheiro Florestal, PhD., Pesquisador da EMBRAPA Cerrados, Caixa Postal 0822, km 18 BR 020, CEP: 73310 970, Planaltina (DF). teodoro@cpac.embrapa.br

Recebido para publicação em 17/07/2004 e aceito em 15/02/2006. 
em cerrado típico (Pereira, 1990). A madeira do gênero Sclerolobium é, sem dúvida, o principal aspecto de suas espécies (Dwyer, 1957; Correia, 1974; Paula, 1986). O termo Sclerolobium vem do grego skleros (duro) e lobium (legume), significando, portanto, "legume duro", numa alusão aos frutos (Barroso et al.1984).

Informações à respeito de como realizar o plantio de Sclerolobium paniculatum utilizando adubação e correção de solo, e a influência dessa prática na qualidade da madeira são escassas. Thomaz (2001) relata a pouca resposta das mudas de Sclerolobium ao fósforo aplicado (fosfato trauíra), não tendo efeito significativo sobre a altura, número de folhas, produção de matéria seca do caule, raiz e parte aérea. Oliveira (2005), trabalhando com a caracterização física da madeira de Sclerolobium paniculatum (carvoeiro), oriunda de plantio homogêneo e adubado encontrou, para a massa específica específica básica, um valor médio de $0,522 \mathrm{~g} / \mathrm{cm}^{3}$ entre cinco níveis de adubação cujas médias não diferiram entre si ao nível de $5 \%$ de probabilidade, havendo, entanto, uma diferenciação quanto à produção de biomassa seca por hectare.

A fertilização pode, segundo alguns autores, influenciar a qualidade da madeira (Hans e Burley, 1972; Brito et al. 1986, Shimoyama e Barrichelo, 1994; Zech e Drechsel 1998). Por outro lado, Zobel (1992) afirma que a aplicação de fertilizantes em pequenas e contínuas doses, em geral, não afeta as características da madeira. Otsamo (1998), estudando o efeito da aplicação de NPK no crescimento de Acacia mangium, Gmelina arborea, Paraseriantes falcataria e Swietenia macrophylla, observou que o fósforo é um elemento essencial para reflorestamentos em biomas dominados por gramíneas. Donald e Schtz (1997) afirmam que, na condução da floresta, a fim de se obter um crescimento uniforme, a adubação é de fundamental importância com conseqüências diretas sobre a indústria florestal.

Vale et al. (2000) não encontraram influência da adubação no poder calorífico de Eucalyptus grandis e Acacia mangium, porém, em Eucalyptus grandis a adubação fosfatada aumentou significativamente a produção energia na forma de calor, por unidade de massa. Estudando o efeito da fertilização na qualidade da madeira de E. grandis aos seis anos de idade, Andrade et al. (1994) encontraram diferenças significativas entre as principais características dendrométricas das árvores.

O presente trabalho tem por objetivo caracterizar a madeira de Sclerolobium paniculatum Vogel var. subvelutinum quanto a algumas de suas propriedades mecânicas e quanto à sua contração, cultivado em plantio homogêneo e sob efeito de diferentes níveis de fertilização com fósforo e cálcio.

\section{MATERIAL E MÉTODOS}

Os dados do estudo foram obtidos de um experimento de fertilização com NPK com a espécie Sclerolobium paniculatum Vogel. var. subvelutinum, conhecida como carvoeiro, instalado em 5/12/1986 em

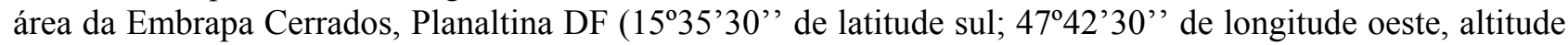
entre 1.000 e $1.025 \mathrm{~m}$ ), num latossolo vermelho-amarelo distrófico. O clima local é tropical com distribuição irregular das chuvas. Os maiores índices pluviométricos são verificados entre os meses de novembro e fevereiro e os menores entre junho e agosto, com precipitação média anual de $1.151 \mathrm{~mm}$.

O delineamento estatístico utilizado foi o de blocos ao acaso, com quatro repetições, cinco tratamentos e parcelas com 25 plantas em espaçamento de $3 \times 2 \mathrm{~m}$, totalizando $6 \mathrm{~m}^{2}$ de área por planta.

O tratamento 1 (T1) é a testemunha. Os outros tratamentos receberam a mesma quantidade de $\mathrm{N}$ (40 $\mathrm{Kg} / \mathrm{ha}), \mathrm{K}(60 \mathrm{~kg} / \mathrm{ha})$ e micronutrientes $(10 \mathrm{~g} / \mathrm{cova})$. A variação ocorreu nas quantidades de fósforo (P). No tratamento T2 foram aplicados $60 \mathrm{~kg}$ de $\mathrm{P}_{2} \mathrm{O}_{5} / \mathrm{ha}$, no T3 $120 \mathrm{~kg}$ de $\mathrm{P}_{2} \mathrm{O}_{5} / \mathrm{ha}$, no T4 $240 \mathrm{~kg}$ de $\mathrm{P}_{2} \mathrm{O}_{5} /$ ha e no tratamento $\mathrm{T} 5$ foram aplicados $120 \mathrm{~kg}$ de $\mathrm{P}_{2} \mathrm{O}_{5} /$ ha acrescido de 1 tonelada de calcário por hectare

Foram abatidas as três árvores de maior circunferência de cada tratamento para a confecção de corpos de prova para os ensaios mecânicos e de contração. Para os ensaios mecânicos de flexão estática, compressão paralela às fibras e cisalhamento foi utilizada a norma MB26 (ABNT, 1940). Foram confeccionados seis corpos de prova por tratamento, perfazendo um total de 30 amostras para cada ensaio. Para determinar as contrações tangencial e radial foi seguida a norma NBR 7190 (1997), partindo de amostras retiradas na posição do diâmetro a altura do peito (DAP) de cada fuste. Todos os ensaios mecânicos e de contração foram realizados na condição saturada.

Os ensaios mecânicos foram realizados em máquina universal de ensaios marca EMIC DL 30000 e programa computacional TESC versão 1.08, no Laboratório de Propriedades Físicas e Mecânicas da Madeira 
da Universidade federal de Lavras, MG. A análise de contração foi realizado no Laboratório de Propriedades Físicas da Madeira na Fazenda Água Limpa/UnB, Brasília, DF.

Os dados obtidos nos ensaios mecânicos de flexão estática, cisalhamento e compressão paralela às fibras foram ajustados para a norma COPANT (Tabela 1), de acordo com o trabalho de Rabelo e Franco (1986).

TABELA 1: Equações ajustadas para algumas características mecânicas cujos valores foram obtidos pela norma COPANT em função de outros obtidos pela proposta de norma MB26 (Rabelo e Franco, 1986).

TABLE 1: Adjusted equations for some mechanical characteristics, which values were obtained in conformity with COPANT convention, in regard to other values obtained by MB26 proposal standard (Rabelo \& Franco, 1986).

\begin{tabular}{l|c|c}
\hline Ensaios & Equações & $\mathrm{r}^{2}$ \\
\hline Flexão estática: & & \\
Resistência máxima $\left(f_{b}\right)$ & $f_{b \text { COPANT }}=63,29+0,801 f_{b \text { ABNT }}$ & 0,83 \\
Módulo de elasticidade $(E)$ & $E_{\text {COPANT }}=27,255+0,583 E_{\text {ABNT }}$ & 0,88 \\
Resistência máxima à compressão axial $\left(f_{c}\right)$ & $f_{c \text { COPANT }}=40,64+0,768 f_{c \text { ABNT }}$ & 0,88 \\
Resistência máxima ao cisalhamento $\left(f_{v}\right)$ & $f_{v \text { COPANT }}=23,41+0,651 f_{v \text { ABNT }}$ & 0,75 \\
\hline
\end{tabular}

Em que: Foram realizadas análises de variância e teste de médias pelo método de Tukey. As análises estatísticas foram realizadas utilizando o programa estatístico SPSS (2000) versão 8.0.

\section{RESULTADOS E DISCUSSÃO}

\section{Flexão estática}

Os resultados dos ensaios de resistência máxima em flexão estática $\left(f_{b}\right)$ e módulo de elasticidade em flexão estática $(E)$ estão apresentados nas Tabelas 2 e 3 e os valores de "F" das análises de variância estão apresentadas na Tabela 3.

TABELA 2: Valores médios da resistência máxima em flexão estática $\left(f_{b}\right)$, para a madeira de Sclerolobium paniculatum Vogel var. subvelutinum, cultivado em plantio homogêneo da EMBRAPA Cerrados, em Planaltina, DF, aos 18 anos de idade, com seus respectivos coeficientes de variação $(\mathrm{CV})$.

TABLE 2: Average values for static bending $\left(f_{b}\right)$ maximum strength of Sclerolobium paniculatum Vogel var. subvelutinum wood 18 years old, cultivated as a homogeneous plantation at EMBRAPA Cerrados, in Planaltina, DF, with respective coefficients of variation (CV).

\begin{tabular}{c|c|c|c|c|c}
\hline Tratamento & N. Amostras & Média $\left(\mathrm{kg} / \mathrm{cm}^{2}\right)$ & CV $(\%)$ & Mínimo $\left(\mathrm{kg} / \mathrm{cm}^{2}\right)$ & Máximo $\left(\mathrm{kg} / \mathrm{cm}^{2}\right)$ \\
\hline T1 & 6 & 645 & 8,5 & 600 & 752 \\
T2 & 6 & 676 & 10,3 & 568 & 736 \\
T3 & 6 & 659 & 7,3 & 608 & 712 \\
T4 & 6 & 640 & 7,2 & 600 & 704 \\
T5 & 6 & 632 & 12,9 & 528 & 776 \\
\hline Total & 30 & 650 & 9,1 & 528 & 776 \\
\hline
\end{tabular}

Em que: $\mathrm{CV}=$ coeficiente de variação. 
TABELA 3: Valores médios para módulo de elasticidade em flexão estática $(E)$ para a madeira de Sclerolobium paniculatum Vogel var. subvelutinum, cultivado em plantio homogêneo da EMBRAPA - Cerrados, em Planaltina, DF, aos 18 anos de idade, com seus respectivos coeficientes de variação $(\mathrm{CV})$.

TABLE 3: Average values for modulus of elasticity $(E)$ in static bending of Sclerolobium paniculatum Vogel var. subvelutinum wood 18 years old, cultivated as a homogeneous plantation at EMBRAPA Cerrados, in Planaltina, DF, with respective coefficients of variation (CV).

\begin{tabular}{cccccccc}
\hline Tratamento & N. Amostras & Média $\left(\mathrm{kg} / \mathrm{cm}^{2}\right)$ & CV $(\%)$ & Mínimo $\left(\mathrm{kg} / \mathrm{cm}^{2}\right)$ & Máximo $\left(\mathrm{kg} / \mathrm{cm}^{2}\right)$ \\
\hline T1 & 6 & 57.398 & 5,8 & 51.839 & 60.370 \\
T2 & 6 & 59.510 & 7,1 & 53.271 & 64.214 \\
T3 & 6 & 62.276 & 7,1 & 57.039 & 67.460 \\
T4 & 6 & 62.037 & 7,0 & 57.081 & 69.759 \\
T5 & 6 & 58.162 & 7,8 & 53.264 & 66.762 \\
\hline Total & 30 & 59.877 & 7,3 & 51.839 & 69.759 \\
\hline
\end{tabular}

Em que: $\mathrm{CV}=$ coeficiente de variação.

Os valores médios de resistência máxima em flexão estática variaram de $632 \mathrm{~kg} / \mathrm{cm}^{2}$ (tratamento T5) a $676 \mathrm{~kg} / \mathrm{cm}^{2}$ (tratamento T2), sendo que dentro do tratamento T5, as amostras apresentaram variação entre $528 \mathrm{~kg} / \mathrm{cm}^{2}$ e $776 \mathrm{~kg} / \mathrm{cm}^{2}$, implicando em coeficiente de variação de 12,9\%. De maneira geral, os coeficientes de variação estão condizentes com a literatura encontrada para ensaios de resistência da madeira (Sales, 1996; Rocco Lahr, 1990). O tratamento T4, que recebeu a maior quantidade de fósforo no experimento (240 $\mathrm{kg} / \mathrm{ha} \mathrm{P}_{2} \mathrm{O}_{5}$ ), apresentou um MOR igual a $640 \mathrm{~kg} / \mathrm{cm}^{2}$, sendo um dos menores valores, superior apenas ao tratamento T5 $\left(632 \mathrm{~kg} / \mathrm{cm}^{2}\right)$, que recebeu $120 \mathrm{~kg} \mathrm{de} \mathrm{P}_{2} \mathrm{O}_{5} /$ ha e mais 1 tonelada de calcário/ha, indicando não haver relação entre a aplicação do fósforo e o módulo de ruptura, comprovada pela análise de variância (Tabela 4), que mostra não haver diferença significativa entre as médias. $O$ valor médio para a resistência máxima em flexão estática foi de $650 \mathrm{~kg} / \mathrm{cm}^{2}$. Os tratamentos $\mathrm{T} 2$ e $\mathrm{T} 3$ apresentaram valores maiores em relação à média geral, enquanto que os tratamentos T1, T4 e T5 apresentaram valores abaixo da média.

O módulo de elasticidade $(E)$ variou de $57.398 \mathrm{~kg} / \mathrm{cm}^{2}$, para a testemunha a $62.276 \mathrm{~kg} / \mathrm{cm}^{2}$ para o tratamento $\mathrm{T} 3$. Os valores para as amostras provenientes de solo adubado foram ligeiramente superiores à testemunha, como pode ser observado pela Tabela 3. As amostras provenientes do tratamento T4, com maior quantidade de fósforo (240 $\mathrm{kg}$ de $\left.\mathrm{P}_{2} \mathrm{O}_{5} / \mathrm{ha}\right)$, apresentaram o segundo maior valor para MOE $\left(62.037 \mathrm{~kg} / \mathrm{cm}^{2}\right)$, abaixo apenas do tratamento T3, que teve aplicação de $120 \mathrm{~kg}$ de $\mathrm{P}_{2} \mathrm{O}_{5} / \mathrm{ha}\left(62.276 \mathrm{~kg} / \mathrm{cm}^{2}\right)$, não havendo diferença significativa ao nível de $5 \%$ de probabilidade, conforme a Tabela 4 . O valor médio para o módulo de elasticidade em flexão estática foi de $59.877 \mathrm{~kg} / \mathrm{cm}^{2}$. Os tratamentos T3 e T4 apresentaram valores médios maiores em relação à média geral, enquanto os tratamentos T1, T2 e T5 apresentam valores abaixo da média.

TABELA 4: Valores de "F" para a resistência máxima $\left(f_{b}\right)$ e para o módulo de elasticidade $(E)$ em flexão estática da madeira de Sclerolobium paniculatum Vogel var. subvelutinum cultivado em plantio homogêneo da EMBRAPA - Cerrados, em Planaltina, DF, aos 18 anos de idade.

TABLE 4: "F" values for maximum strength $\left(f_{b}\right)$ and modulus of elasticity $(E)$ in static bending of Sclerolobium paniculatum Vogel var. subvelutinum wood 18 years old, cultivated as a homogeneous plantation at EMBRAPA - Cerrados, in Planaltina, DF.

\begin{tabular}{l|c|c|c}
\hline Variável & Graus de & \multicolumn{2}{|c}{ Valores de "F" } \\
\cline { 3 - 4 } & Liberdade & $f_{b}$ & $E$ \\
\hline Entre Grupos & 4 & & $1,683^{\text {ns }}$ \\
Intra Grupos & 25 & $0,472^{\text {ns }}$ & \\
Total & 29 & & \\
\hline
\end{tabular}

Gray e Kyanka (1974), trabalhando com a madeira Pinus resinosa adubado com potássio, também observaram comportamento semelhante para $f_{b}$ e $E$. Brito et al. (1986), analisando a madeira de Pinus caribaea var. bahamensis oriunda de plantio adubado, observaram alterações na resistência da madeira, fato também observado em E. gandis por Valeri et al (1991). Nyakuengama et al. (2004) encontraram que a adubação promoveu aumento da produção de biomassa sem efeitos adversos sobre as propriedades da 
madeira de Pinus radiata.

\section{Cisalhamento e compressão axial}

Os resultados do ensaio de resistência máxima ao cisalhamento $\left(f_{v}\right)$ e compressão axial $\left(f_{c}\right)$ estão apresentados nas Tabelas 5 e 6 e os valores de "F" das análises de variância estão apresentados na Tabela 7. A resistência máxima ao cisalhamento (Tabela 5) foi inferior ao valor médio encontrado para a testemunha $\left(143 \mathrm{~kg} / \mathrm{cm}^{2}\right)$, sendo que o menor valor foi encontrado para o tratamento T5 $\left(125 \mathrm{~kg} / \mathrm{cm}^{2}\right)$. O tratamento T3 apresentou o maior coeficiente de variação (15,6\%), com uma amplitude de $118 \mathrm{~kg} / \mathrm{cm}^{2}$ a $147 \mathrm{~kg} / \mathrm{cm}^{2}$. Apesar dos valores absolutos mostrarem uma tendência de diminuição da resistência máxima ao cisalhamento, a análise de variância mostra não haver diferença significativa entre as médias dos tratamentos ao nível de 5\% de probabilidade. O valor médio para a resistência máxima ao cisalhamento foi de $131 \mathrm{~kg} / \mathrm{cm}^{2}$.

TABELA 5: Valores médios para resistência máxima ao cisalhamento para a madeira de Sclerolobium paniculatum Vogel var. subvelutinum cultivado em plantio homogêneo na EMBRAPA Cerrados, em Planaltina, DF, aos 18 anos de idade, com seus respectivos coeficientes de variação $(\mathrm{CV})$.

TABLE 5: Average values for maximum shearing strength of Sclerolobium paniculatum Vogel var. subvelutinum wood 18 years old, cultivated as a homogeneous plantation at EMBRAPA Cerrados, in Planaltina, DF, with respective coefficients of variation (CV).

\begin{tabular}{cccccccc}
\hline Tratamento & N. amostras & Média & CV $(\%)$ & Mínimo & Máximo \\
\hline T1 & 2 & 143 & 10,0 & 133 & 153 \\
T2 & 6 & 132 & 6,8 & 117 & 140 \\
T3 & 2 & 132 & 15,6 & 118 & 147 \\
T4 & 6 & 132 & 4,3 & 121 & 138 \\
T5 & 6 & 125 & 9,9 & 105 & 133 \\
\hline Total & 22 & 131 & 8,3 & 105 & 153 \\
\hline
\end{tabular}

TABELA 6: Valores médios para resistência máxima à compressão paralela às fibras para a madeira de Sclerolobium paniculatum Vogel var. subvelutinum cultivado em plantio da EMBRAPA Cerrados, em Planaltina, DF, aos 18 anos de idade, com seus respectivos coeficientes de variação $(\mathrm{CV})$.

TABLE 6: Average values for maximum compression parallel-to-grain strength of Sclerolobium paniculatum Vogel var. subvelutinum wood 18 years old, cultivated as a homogeneous plantation at EMBRAPA - Cerrados, in Planaltina, DF, with respective coefficients of variation (CV).

\begin{tabular}{c|c|c|c|c|c}
\hline Tratamento & N. amostras & Média & CV (\%) & Mínimo & Máximo \\
\hline T1 & 6 & 309 & 12,4 & 241 & 356 \\
T2 & 6 & 284 & 9,7 & 250 & 314 \\
T3 & 6 & 306 & 12,3 & 266 & 357 \\
T4 & 6 & 294 & 5,0 & 281 & 322 \\
T5 & 6 & 287 & 6,5 & 262 & 312 \\
\hline Total & 30 & 296 & 9,7 & 241 & 357 \\
\hline
\end{tabular}

A compressão axial (Tabela 6) apresentou média de $296 \mathrm{~kg} / \mathrm{cm}^{2}$, não havendo diferença significativa ao nível de $5 \%$ de probabilidade entre tratamentos. Observa-se que os valores de amostras provenientes de solo adubado foram inferiores àqueles encontrados para a testemunha, com o tratamento $\mathrm{T} 2$ apresentando $\mathrm{o}$ menor valor para a resistência máxima à compressão axial $\left(284 \mathrm{~kg} / \mathrm{cm}^{2}\right)$. O tratamento T4 e T5 apresentaram valores médios de 294 e $287 \mathrm{~kg} / \mathrm{cm}^{2}$ respectivamente. O valor médio de resistência máxima à compressão axial foi de $296 \mathrm{~kg} / \mathrm{cm}^{2}$.

Resultados semelhantes foram encontrados por Zobel (1992), que afirma não haver diferenças na qualidade da madeira oriunda de plantios adubados, fato também observado por Valeri et al. (1991) trabalhando com Eucalyprus grandis e por Nyakuengama et al. (2004) estudando a influência da adubação em Pinus radiata na Austrália. 
TABELA 7: Valores de "F" para a resistência máxima ao cisalhamento e à compressão axial, da madeira de Sclerolobium paniculatum Vogel var. subvelutinum cultivado em plantio homogêneo da EMBRAPA - Cerrados, em Planaltina, DF, aos 18 anos de idade.

TABLE 7: "F" values for maximum shear strength and for maximum axial compression strength of Sclerolobium paniculatum Vogel var. subvelutinum wood 18 years old, cultivated as a homogeneous plantation at EMBRAPA - Cerrados, in Planaltina, DF.

\begin{tabular}{l|c|c|c}
\hline Variável & Graus de & \multicolumn{2}{|c}{ Valores de "F" } \\
\cline { 3 - 4 } & Liberdade & $f_{v}$ & $f_{c}$ \\
\hline Entre Grupos & 4 & & $0,900^{\mathrm{ns}}$ \\
Intra Grupos & 17 & $1,185^{\mathrm{ns}}$ & \\
Total & 21 & & \\
\hline
\end{tabular}

\section{Contração volumétrica, tangencial e radial}

A Tabela 8 apresenta os valores das contrações para a madeira de Sclerolobium paniculatum com seus respectivos coeficientes de variação.

TABELA 8: Massa Específica Básica e Contração da Madeira de Sclerolobium paniculatum Vogel var. subvelutinum cultivado em plantio homogêneo da EMBRAPA - Cerrados, em Planaltina, DF, aos 18 anos de idade.

TABLE 8: Specific Gravity and Shrinkage of Sclerolobium paniculatum Vogel var. subvelutinum wood 18 years old, cultivated as a homogeneous plantation at EMBRAPA - Cerrados, in Planaltina, DF.

\begin{tabular}{|c|c|c|c|c|c|c|}
\hline \multirow{2}{*}{\multicolumn{2}{|c|}{ Tratamentos }} & \multirow{2}{*}{$\begin{array}{l}\text { Massa específica } \\
\text { básica }\left(\mathrm{g} / \mathrm{cm}^{3}\right)^{*}\end{array}$} & \multicolumn{3}{|c|}{ Contrações (\%) } & \multirow{2}{*}{ Relação T/R } \\
\hline & & & Tangencial & Radial & Volumétrica & \\
\hline \multirow{2}{*}{$\mathrm{T} 1$} & Média & 0,52 & 6,6 & 3,32 & 10,35 & \multirow{2}{*}{1,99} \\
\hline & $\mathrm{CV}$ & $3,33 \%$ & $16 \%$ & $4 \%$ & $10,40 \%$ & \\
\hline \multirow{2}{*}{$\mathrm{T} 2$} & Média & 0,52 & 7,36 & 3,56 & 11,54 & \multirow{2}{*}{2,04} \\
\hline & $\mathrm{CV}$ & $0,96 \%$ & $5 \%$ & $9 \%$ & $7,2 \%$ & \\
\hline \multirow{2}{*}{$\mathrm{T} 3$} & Média & 0,56 & 7,53 & 3,26 & 11,27 & \multirow{2}{*}{2,31} \\
\hline & $\mathrm{CV}$ & $5,46 \%$ & $11 \%$ & $7 \%$ & $9,64 \%$ & \\
\hline \multirow{2}{*}{$\mathrm{T} 4$} & Média & 0,51 & 7,38 & 2,67 & 10,50 & \multirow{2}{*}{2,76} \\
\hline & $\mathrm{CV}$ & $8,07 \%$ & $11 \%$ & $18 \%$ & $10,38 \%$ & \\
\hline \multirow{2}{*}{ T5 } & Média & 0,50 & 7,13 & 3,17 & 10,85 & \multirow{2}{*}{2,24} \\
\hline & $\mathrm{CV}$ & $3,72 \%$ & $8 \%$ & $11 \%$ & $6,30 \%$ & \\
\hline \multirow[t]{2}{*}{ Média } & Geral & 0,52 & 7,2 & 3,2 & 10,90 & 2,25 \\
\hline & $\mathrm{CV}$ & 4,2 & 5,8 & 11,8 & 5,3 & 13,5 \\
\hline
\end{tabular}

Em que: * = Oliveira (2005); CV = coeficiente de variação; $\mathrm{T}$ = contração tangencial; $\mathrm{R}$ = contração radial.

Observa-se, pela Tabela 9, não haver relação entre a aplicação de fósforo e as contrações da madeira de Sclerolobium paniculatum. O tratamento T4 apresentou valores médios intermediários $(7,38 \%$ de contração tangencial, $2,67 \%$ de contração radial e $10,50 \%$ de contração volumétrica) contra valores máximos (contração tangencial de 7,53\% para o tratamento T3, contração radial 3,56\% e contração volumétrica de $11,54 \%$ ) para o tratamento T2. A contração volumétrica média foi de $10,90 \%$, caracterizando a madeira como de baixa contração, segundo o IPT (1985). A razão entre a contração tangencial e radial (T/R) indica a tendência da madeira apresentar defeitos relacionados a sua secagem (Oliveira e Silva, 2003; Scavanaca Junior e Garcia, 2004). A madeira de Sclerolobium paniculatum apresentou uma média para a relação T/R de 2,25 e amplitude de 1,99 a 2,76 para os tratamentos T1 e T4 respectivamente. Não foram observadas diferenças significativas entre as médias de contração da madeira de Sclerolobium paniculatum (Tabela 8). 
TABELA 9: Valores de "F" para a contração tangencial e radial da madeira de Sclerolobium paniculatum Vogel var. subvelutinum cultivado em plantio homogêneo da EMBRAPA - Cerrados, em Planaltina, DF, aos 18 anos de idade.

TABLE 9: "F" values for tangential and radial shrinkage of Sclerolobium paniculatum Vogel var. subvelutinum wood 18 years old, cultivated as a homogeneous plantation at EMBRAPA Cerrados, in Planaltina, DF.

\begin{tabular}{l|l|c|c}
\hline \multirow{2}{*}{ Variável } & \multirow{2}{*}{ Graus de Liberdade } & \multicolumn{2}{|c}{ Valores de "F" para as contrações } \\
\cline { 3 - 4 } & & Tangencial & Radial \\
\hline Entre Grupos & 4 & & \\
Intra Grupos & 10 & 0,675688 & 3,224332 \\
Total & 14 & & \\
\hline
\end{tabular}

A Figura 1 ilustra a variação das contrações radial, tangencial e volumétrica entre os tratamentos.

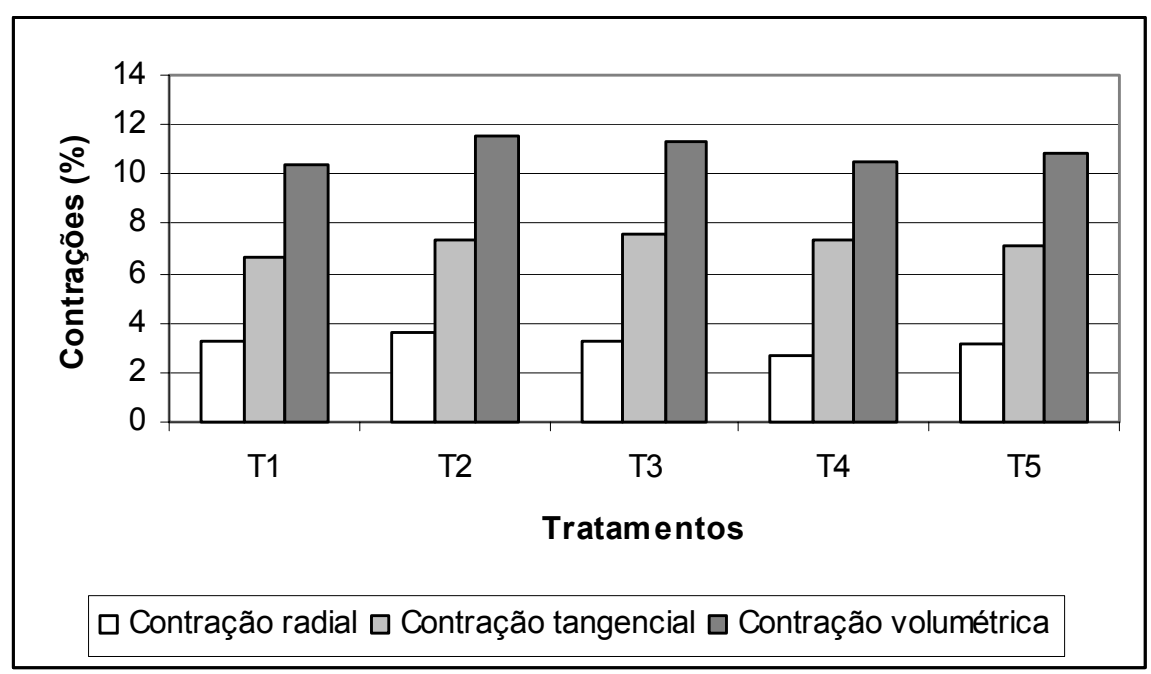

FIGURA 1: Contrações radiais, tangenciais e volumétricas para a madeira de Sclerolobium paniculatum Vogel var. subvelutinum cultivado em plantio homogêneo na EMBRAPA - Cerrados, Planaltina, DF, aos 18 anos de idade.

FIGURE 1: Radial, tangential and volumetric shrinkages of Sclerolobium paniculatum Vogel var. subvelutinum wood 18 years old, cultivated as a homogeneous plantation at EMBRAPA Cerrados, in Planaltina, DF.

\section{CONCLUSÕES}

Considerando os resultados obtidos nesta pesquisa, pode-se concluir que, para a madeira de Sclerolobium paniculatum var. subvelutinum (carvoeiro) utilizado no estudo:

Os diferentes níveis de adubação aplicados em plantio homogêneo de Sclerolobium paniculatum var. subvelutinum não interferiram, de forma significativa, nas propriedades mecânicas e de contração da madeira dessa espécie.

Apesar dos resultados não-significativos em nível estatístico, o módulo de elasticidade para flexão estática mostra tendência de aumentar os valores, em termos absolutos, e o módulo de ruptura mostra tendência de diminuir os valores, também em termos absolutos, conforme o nível de adubação.

No caso das propriedades mecânicas cisalhamento e compressão paralela às fibras, a madeira dos tratamentos, que receberam adubação, mostra tendência de apresentar módulos de rupturas menores do que aqueles da testemunha.

As árvores de Sclerolobium paniculatum var. subvelutinum em plantios homogêneos apresentam-se com muitos galhos. Dessa forma, o crescimento deve ser conduzido de maneira a produzir madeira com quantidade menor de nós por meio de tratos culturais, como a retirada sistemática de galhos. 


\section{AGRADECIMENTO}

Os autores agradecem ao professor José Tarcísio Lima da área de Tecnologia da Madeira do Departamento de Ciências Florestais da Universidade Federal de Lavras (UFLA) pela ajuda na obtenção dos dados das características mecânicas.

\section{REFERÊNCIAS BIBLIOGRÁFICAS}

ANDRADE, A. M.; VITAL, B.R.; BARROS, N.F.; DELlA LUCIA, R. M.; CAMPOS, J.C.C.; VALENTE, O.F. Efeitos da fertilização mineral e da calagem do solo na produção e na qualidade da madeira de eucalipto. Revista Árvore, v. 18, n.1, p. 69-78, 1994.

ASSOCIAÇÃO BRASILEIRA DE NORMAS TÉCNICAS. Ensaios físicos e mecânicos de madeiras. Método brasileiro, MB-26. Rio de Janeiro : ABNT, 1940. (MB26).

ASSOCIAÇÃO BRASILEIRA DE NORMAS TÉCNICAS. Projeto de estruturas de madeira. Rio de Janeiro : ABNT, 1997. (NBR-7190).

BARROSO, G. M.; PEIXOTO, A. R.; ICHSO, C. L.; COSTA, C. G.; GUIMARÃES, E. F.; LIMA H. C. Sistemática de angiospermas do Brasil. Viçosa : Imp. Universitária, 1984. v.2. 377 p.

BRITO, J.O.; FERRAZ, E. S. B.; BARRICHELLO, L.E. G. A adubação mineral e seus efeitos sobre os anéis de crescimento da madeira de Pinus caribaea var. bahamensis. IPEF, v.32, p.5-17, 1986.

CHÉVEZ POZO, O. V. O Pequi (Caryocar brasiliense) : uma alternativa para desenvolvimento sustentável do cerrado do norte de Minas Gerais. 1997. 100p. Dissertação (Mestrado em Administração Rural) - Universidade Federal de Lavras, Lavras, 1997.

CORREIA, M. P. Dicionário das plantas úteis do Brasil e das exóticas cultivadas. Rio de Janeiro : Ministério da Agricultura : IBDF, 1974. v. 1. p. 44-109.

DONALD, D. E. M; SCHTZ, C. J. The response of Eucalyptus to fertilizer application at planting: the Low's Creek Trial. South African Forestry Journal, Pretoria, n. 102, p. 23-28, 1977.

DWYER, J. D. The tropical genus Sclerolobium Vogel (Caesalpinaceae). Lloydia, Cincinati, v. 20, n. 2, p. 67-118, 1957.

GRAY, R. L.; KYANKA, G. H. Potassium fertilization effects on the static bending properties of red pine wood. Forest Products Journal, v. 24, n. 9, p. 92-96, 1974.

HANS, A. S.; BURLEY, U. Wood quality of Eucalyptus grandis Hill ex. Maiden in a fertilizer trial at Siamambo, Zambia. East African Agricultural and Forestry Journal, Nairobi, v. 38, n. 2, p. 157-161, 1972.

INSTITUTO DE PESQUISAS TECNOLÓGICAS. Madeira: o que é e como pode ser processada e utilizada. Boletim ABPN, n. 36, p. 1-189, 1985.

KLINK, C. A. Relação entre o desenvolvimento agrícola e a biodiversidade. In: VIII SIMPÓSIO SOBRE O CERRADO, 8., 1996, Brasília ; INTERNATIONAL SYMPOSIUM ON TROPICAL SAVANNAS, 1., 1996, Brasília. Anais... Brasília, 1996.

NYAKUENGAMA, J. G.; DOWNS, G. M.; NAMBIAR, E. K. S.; BLAKEMORE, P.; NORTHWAY, R. N. Effect of later age stand management on quality of radiata pine wood : Project No. PN97.403. Victoria : Forest and Wood Products Research and Development Corporation, 2004.

OLIVEIRA, J.T. S.; SILVA, J. C. Radial variation of shirinkage and specific gravity in Eucalyptus saligna Sm. wood. Rev. Árvore, v. 27, n. 3, p. 381-385, 2003.

OLIVEIRA, I.R.M. Características da madeira e rendimento em biomassa de um plantio homogêneo de Sclerolobium paniculatum com diferentes níveis de adubação. 39p. 2005. Dissertação (Mestrado em Engenharia Florestal) - Universidade de Brasília, Brasília, 2005. (Publicação EFL - 048/2005, Departamento de Engenharia Florestal, DF)

OTSAMO, A. Effect of fertilizing on estabelishment and early Growth of tree plantation on Imperata cylindrica grassland. In: Autor. Soils of tropical forest ecosystems: characteristics, ecology and management. New York : Springer, 1998. p. 137-143.

PAULA, J.E. Estudo dendrométrico e anatômico de Sclerolobium densiflorum Benth visando seu aproveitamento na produção de energia. In: CONGRESSO NACIONAL DE BOTÂNICA, 37, 1986, Ouro Preto. Resumos. Ouro Preto, Sociedade Botânica do Brasil, 1986. p. 69. 
PEREIRA, B. A. S. Estudo morfo-anatômico da madeira, casca, e folha de duas variedades vicariantes de Sclerolobium paniculatum Vogel (Leguminosae, Casalpinioideae) de mata e cerrado. 1990. 198p. Dissertação (Mestrado) - Escola Superior de Agricultura Luiz de Queirós, Piracicaba, 1990.

RABELO, M.; FRANCO, N. Estudo comparativo entre os métodos para o ensaio de madeira apresentados nas normas COMPANT e ABNT. São Paulo: IPT, 1986. 18 p.

ROCCO LAHR, F. A. Considerações a respeito da variabilidade de propriedade de resistência e de elasticidade da madeira. 1990. _ _ p. Tese (Livre Docência) - Escola de Engenharia de São Carlos, Universidade de São Paulo, São Carlos, 1990. p. 5-104.

RODRIGUES, L. A.; CARVALHO, A C.; GOMES, J. L.; BOTREL, R. T. Espécies vegetais nativas usadas pela população local em Luminárias, MG. Boletim agropecuario da Universidade Federal de Lavras, n. 52, p.1-34, 2002. SALES, A. Proposição de classes de resistência para madeira. 1996. 223p. Tese (Doutorado) - Escola Politécnica de São Paulo, São Paulo, 1996.

SCAVANACA JUNIOR, L.; GARCIA, J. N. Determinação das propriedades físicas e mecânicas da madeira de Eucalyptus urophylla. Scientia Forestalis, n. 65, p.120-129, 2004.

SHIMOYAMA, V.R. S.; BARRICHELO, L.E.G. Importância da adubação na qualidade da madeira e celulose. In: SÁ, Marco Eustáquio de e BUZZETI, Salatier Coordenadores. Importância da adubação da qualidade dos produtos agrícolas. São Paulo: Icone, 1994. p. 153-160.

STATISTICAL Package for the Social Sciences, SPSS. Versão 8.0 for Windows. 2000.

THOMAZ, M. A. A.; VIÉGAS, I. J. M.; SAMPAIO, M.C.T.; MATOS, A. O.; CONCEIÇÃO, H.E.O. Crescimento de plantas jovens de taxi-branco (Sclerolobium paniculatum Vogel) em função da idade de aplicação e fosfato de trauíra. Revista Ciência Agrária, Belém, n. 35, p. 15-24, 2001.

VAlE, A.T.; BRASIL, M.A.M.; CARVALGO, C.M.; VEIGA, R. A. A. Produção de energia do fuste de Eucalyptus grandis Hill ex. Maiden e Acacia mangium Willd em diferentes níveis de adubação. Revista Cerne, v. 6, n. 1, p. 33-88, 2000 .

VALERI, S.V.; CORRADINI, L.; AGUIAR, I.V. Efeitos de níveis de NPK e calcário dolomítico na produção volumétrica de madeira de Eucalyptus grandis Hill ex Maiden. Científica, v. 19, n. 1, p. 63-70, 1991.

ZECH, W.; DRECHSEL, P. Nutrient disorders and nutrient management in fast growing plantations. In: Soils of tropical forest ecosystems: characteristics, ecology and management. New York : Springer, 1998, p. 99-105.

ZOBEL, B. Silvicultural effects on wood properties. IPEF International, v. 2, p. 31-38, 1992. 